\title{
INVARIANT IDEALS OF COMMUTATIVE RINGS
}

\author{
by ROBERT L. SNIDER
}

(Received 11 November, 1991; revised 4 May, 1992)

Let $R$ be a commutative Noetherian ring and $G$ a group of elements acting on $R$ as automorphisms. In this note, we are concerned with the structure of the lattice of invariant ideals of $R$. In particular we shall compute the Krull dimension of this lattice. Our group is an arbitrary group. There are none of the usual assumptions of some sort of algebraic action.

By Krull dimension, we mean the notion of Krull dimension introduced by Rentschler and Gabriel [1, p. 180]. This definition attaches an ordinal number to a Noetherian lattice (and to certain other lattices). (For a definition, see below.) For a commutative Noetherian ring, the Krull dimension of the lattice of ideals is the same as the usual (classical) Krull dimension in terms of the longest chain of prime ideals $[1, p$. 192]. It follows that in some sense the structure of the lattice of ideals is determined by the poset of prime ideals. The point of this paper is that the same holds for the lattice of invariant ideals. However there may not be enough invariant prime ideals but there are enough prime ideals with finite orbit. We recall that an ideal with finite orbit is called an orbital ideal [2].

The Krull dimension of a lattice can be an infinite ordinal. One can define the classical Krull dimension so that the classical Krull dimension can be an infinite ordinal [1, p. 191]. (For a definition, see below.) We will denote the Krull dimension of the lattice of invariant ideals by $\kappa_{G}(R)$ and the classical Krull dimension of the poset of orbital primes by $d_{G}(R)$. Thus if there is a bound on the lengths of chains of prime ideals, $d_{G}(R)$ is the maximum length of a chain. If there is no bound, then $d_{G}(R)$ will be an infinite ordinal.

THEOREM 1. If $R$ is a commutative Noetherian ring with a group $G$ acting on it, then $\kappa_{G}(R)=d_{G}(R)$.

An invariant ideal $P$ of $R$ is called $G$-prime if whenever $I J P$ for invariant ideals $I$ and $J$, then $I \subseteq P$ or $J \subseteq P$. Theorem 1 can be restated using chains of $G$-prime ideals as follows.

THEOREM 2. If $R$ is a commutative Noetherian ring with a group $G$ acting on it, then $\kappa_{G}(R)$ is the classical Krull dimension of the poset of $G$-prime ideals of $R$.

Let $A$ be a free abelian group of rank $n$ and $k$ a field. If $G$ is a subgroup of $\mathrm{GL}_{n}(Z)$, then $G$ acts on $A$ and hence on the group ring $k[A]$. In this case the length of the longest chain of orbital primes of $k[A]$ has already been calculated. In order to state this, we recall some definitions [2]. A non-identity orbital subgroup $B$ of $A$ is a plinth if $Q \otimes_{Z} B$ contains no nontrivial orbital subspaces. If $B$ is a plinth we can replace it with its pure closure. Then a subgroup $H$ of finite index acts on $B$ and hence on the free abelian group $A / B$. In this way we can define a chain of subgroups $B_{1} \subseteq B_{2} \subseteq \ldots \subseteq B_{n}=A$ such that $B_{i+1} / B_{i}$ is a plinth in $A / B_{i}$. The integer $n$ is called the plinth length of $A$. Roseblade has shown that the plinth length of $A$ is the same as the length of the longest chain of orbital prime ideals of $k[A][2, \mathrm{p} .437]$. Hence we have the following corollary. 
COROLlaRY 1. Let $G$ act on the finitely generated free abelian group $A$ and hence on the group ring $k[A]$. The Krull dimension of the lattice of $G$-invariant ideals of $k[A]$ is the plinth length of $A$.

As the definition of the Krull dimension of a lattice may be unfamiliar to commutative ring theorists, we recall the definition [1, p. 174]. Let $L$ be a lattice. If it is trivial, we say $\kappa(L)=-\infty$. If $L$ has DCC and is nontrivial, then $\kappa(L)=0$. For a general ordinal $\alpha$, we define $\kappa(L)=\alpha$ provided (i) $\kappa(L) \neq \beta<\alpha$ and (ii) in any descending chain of elements of $L$, all but finitely many factors (intervals) have Krull dimension less than $\alpha$. Lattices with ACC will always have a Krull dimension.

We recall the definition of the dimension of a poset $A$ satisfying ACC [1, p. 191] (not the Krull dimension!). Let $A_{0}$ be the subset of maximal elements of $A$; and, for each ordinal $\alpha$, let

$$
A_{\alpha}=\left\{a \in A: b \in A, b>a \text { implies } b \in A_{\beta} \text { for some } \beta<\alpha\right\} .
$$

Then $\operatorname{dim} A$ is the least $\alpha$ with $A_{\alpha}=A$. By the classical Krull dimension of a set $\mathscr{P}$ of prime ideals (or $G$-prime ideals), we shall mean the dimension of the poset $\mathscr{P}$.

The equivalence of Theorems 1 and 2 follow from the next lemma.

Lemma 1. The classical Krull dimension of the lattice of orbital prime ideals is the same as the classical Krull dimension of the G-invariant primes.

Proof. Let $A$ be the set of orbital primes and let $A_{0}$ be the maximal orbital prime ideals and, inductively for the ordinal $\alpha$, let

$$
A_{\alpha}=\left\{P \in A: Q \in A, Q>P \text { implies } Q \in A_{\beta} \text { for some } \beta<\alpha\right\} \text {. }
$$

Similarly define $B_{\alpha}$ for the $G$-invariant ideals. If $P$ is an orbital prime, then $\cap P^{g}$ is a $G$-prime ideal. Furthermore all $G$-prime ideals $Q$ arise this way. (The minimal primes over $Q$ are orbital and, if $P$ is any such one, $\cap P^{g}=Q$.) Our proof will be by showing that $P \in A_{\alpha}$ if and only if $\cap P^{g} \in B_{\alpha}$. We induct on $\alpha$. For $\alpha=0$, let $P \in A_{0}$. If $\cap P^{g} \subseteq \bigcap Q^{g}$, where $P$ and $Q$ are orbital primes, then, for some $g$ and $h, P^{g} \subseteq Q^{h}$. But $P^{g}$ is maximal; so $P^{g}=Q^{h}$. It follows that $\bigcap P^{g}$ is in $B_{0}$. Conversely, suppose $\bigcap P^{g}$ is in $B_{0}$ and $P \subseteq Q$, where $Q$ is orbital. Then $\cap P^{g}=\bigcap Q^{g}$. But the orbit of $P$ and the orbit of $Q$ both consist of the minimal primes over $\cap P^{8}$. Hence $P=Q$ and $P \in A_{0}$. Now inductively suppose the statement is true for all ordinals less than $\alpha$. Suppose that $P \in A_{\alpha}$. If $\cap P^{g} \subsetneq Q^{g}$, then, for some $h, P \subseteq Q^{h}$. Furthermore $P \neq Q^{h}$ since otherwise $\cap P^{g}=\cap Q^{g}$. Hence $Q^{h}$ is in $A_{\beta}$ and by induction $\cap Q^{g}$ is in $B_{\beta}$. It follows that $\cap P^{g} \in B_{\alpha}$. Conversely, suppose that $\cap P^{g} \in B_{\alpha}$. Suppose $Q$ is an orbital prime and that $P \subsetneq Q$. Then $\cap P^{g} \subset \cap Q^{g}$. If they were equal, then the orbit of $P$ and the orbit of $Q$ both would consist of the minimal primes over $\cap P^{g}$. Then $P=Q$, a contradiction. Therefore $\cap Q^{g} \in B_{\beta}$ and by induction $Q \in A_{\beta}$. It follows that $P \in A_{\alpha}$.

Our original proof was to prove Theorem 1 directly using the orbital primes. The following proof was suggested to us by the referee. It involves a notion of $G$-primary decomposition which may be well known.

Let $R$ be a commutative Noetherian ring and let $G$ be a group acting on $R$ as automorphisms. An invariant ideal will be called a $G$-ideal. A $G$-ideal $I$ is $G$-primary if whenever $A$ and $B$ are $G$-ideals with $A B \subseteq I$, then either $A \subseteq I$ or $B^{n} \subseteq I$ for some $n$. A 
$G$-ideal $I$ is $G$-irreducible if whenever $A$ and $B$ are $G$-ideals with $A \cap B=I$, then either $A=I$ or $B=I$.

Lemma 2. If $I$ is a G-ideal of $R$, then $\operatorname{rad}(I)$ is a $G$-ideal. Furthermore if $I$ is $G$-primary, then $\operatorname{rad}(I)$ is $G$-prime.

Proof. Obviously $\operatorname{rad}(I)$ is $G$-invariant. Let $P$ be $\operatorname{rad}(I)$. Suppose $I$ is $G$-primary. If $A$ and $B$ are $G$-ideals with $A B \subseteq P$, then since, for some $n, P^{n} \subseteq I$, we must have $(A B)^{n}=A^{n} B^{n} \subseteq I$. If $A \nsubseteq P$, then $A^{n} \ddagger P$ and hence $A^{n} \ddagger I$. Therefore $B^{n m} \subseteq I$ and hence $B \subseteq P$.

Lemma 3. Any G-irreducible is G-primary.

Proof. Let $I$ be $G$-irreducible and suppose $A B \subseteq I$ for $G$-ideals $A$ and $B$. We may assume $I \subseteq A$ and $I \subseteq B$. Suppose that $A \nsubseteq I$. By the Artin-Rees lemma $A \cap B^{n} \subseteq A B \subseteq I$ for some $n$ and hence $A \cap\left(B^{n}+I\right)=A \cap B^{n}+I \subseteq A B+I=I$. Since $A$ and $B^{n}+I$ are $G$-ideals and $I$ is $G$-irreducible and $A \neq I$, we have $B^{n}+I=I$ and hence $B^{n} \subseteq I$.

Corollary 2. Any G-ideal has a G-primary decomposition.

Lemma 4. Let I be a $G$-primary ideal with radical $P$. Then there is DCC on $G$-primary ideals between $I$ and $P$.

Proof. Let $Q$ be a prime ideal minimal over $P$, then the (finite) orbit of $Q$ consists of all the minimal primes over $P$ and $P=\cap Q^{g}$. Let $S=R-\cap Q^{g}$. We claim that if $J$ is a $P$-primary ideal then $J=J R_{S} \cap R$. Let $X=J R_{S} \cap R$. If $x \in X$, then $x=j / s$ for some $j \in J$ and $s \in S$. It follows that $x s=j$. Now $R$ is a Noetherian ring so $X$ is finitely generated. Hence there exists a single $s$ with $X s \subseteq J$. Let $Y=\{r: X r \subseteq I\}$. Now $X Y \subseteq J$ and $X$ and $Y$ are $G$-ideals. If $X \nsubseteq J$, then $Y^{n} \subseteq J$ which implies $s^{n} \in J$, a contradiction. Now $P^{n} \subseteq I$ for some $n$ and $\left(P R_{S}\right)^{i} /\left(P R_{S}\right)^{i+1}$ is a finitely generated module over the Artinian ring $R_{S} / P R_{S}$.

Lemma 5. If $Q_{1} \cap A \subseteq Q_{2}$, where $Q_{1}$ and $Q_{2}$ are $G$-primary and $A$ is a $G$-ideal and $A^{n} \ddagger Q_{2}$ for all $n$, then $Q_{1} \subseteq Q_{2}$.

Proof. $Q_{1} A \subseteq Q_{2}$, so if $Q_{1} \nsubseteq Q_{2}$, then $A^{n} \subseteq Q_{2}$, a contradiction.

We recall that a module $M$ is $G$-critical if $\kappa_{G}(M)=\alpha$ and $\kappa_{G}(M / I)<\alpha$ for all nonzero $G$-submodules $I$.

Let $P$ be a $G$-prime ideal. Let $Q$ be a minimal prime over $P$. Let $S=R-\cap Q^{g}$. We form $R_{S}$ and define the symbolic powers $P^{(n)}=R \cap P^{h} R_{S}$.

LeмmA 6. If 0 is a G-primary ideal and $P$ is a G-prime ideal, then $\cap P^{(n)}=0$.

Proof. The usual commutative proof works.

Lemma 7. Let $P$ be a $G$-prime ideal with $\kappa_{G}(R / P)=\alpha$. Then (i) $R / P$ is $G$-critical, and (ii) if $I \varsubsetneqq J$ are $G$-ideals with $I P$-primary, then $\kappa_{G}(J / I)=\alpha$.

Proof. Suppose the result fails, but, by Noetherian induction holds for all $G$-prime ideals bigger than $P$. First suppose $Q$ is a $G$-prime and $P \subsetneq Q$. For the moment assume that $P=0$. Letting $Q^{(n)}$ denote the symbolic powers, we have $\cap Q^{(n)}=0$. Now each $Q^{(n)}$ is $Q$-primary and hence by induction $\kappa_{G}\left(Q^{(n)} / Q^{(n+1)}\right)=\kappa_{G}(R / Q)=\beta$. Since there are infinitely many such factors we must have $\beta<\alpha$. By Lemma 4 and the Noetherian 
condition, there is a saturated chain of $P$-primary ideals $R=I_{0} \supseteq P=I_{1} \supseteq I_{2} \supseteqq \ldots \supseteqq I_{m}=I$ between $R$ and $I$. It is enough to show that $I_{n} / I_{n+1}$ is $\alpha-G$-critical. Let $J$ be a $G$-ideal with $I_{n+1} \subsetneq J \subseteq I_{n}$. Note that the $P$-primary component of $J$ is $I_{n}$, so suppose that $J=I_{n} \cap Q_{1} \cap \ldots \cap Q_{s}$ is an irredundant $G$-primary decomposition of $J$. There is a strictly increasing map from $\mathscr{L}\left(I_{n} / J\right)$, the lattice of $G$-ideals between $I_{n}$ and $J$, to $\mathscr{L}(R / Q) \times$ $\ldots \times \mathscr{L}\left(R / Q_{s}\right)$ and so $\kappa_{G}\left(I_{n} / J\right) \leq \max \kappa_{G}\left(R / Q_{i}\right)$. Now if $P_{i}=\sqrt{Q_{i}}$, then $P \varsubsetneqq P_{i}$ since some power of $P$ is contained in $I_{n+1} \subseteq Q_{i}$. Since the result holds for each $P_{i}$, $\kappa_{G}\left(R / Q_{i}\right)<\alpha$ and so $\kappa_{G}\left(I_{n} / J\right)<\alpha$ for $n \geq 1$.

It remains to show that $\kappa_{G}\left(I_{n} / I_{n+1}\right) \geq \alpha$ for $n \geq 1$. We do this by showing that $\kappa_{G}\left(I_{n} / I_{n+1}\right)>\beta$ for all $\beta<\alpha$. Without loss of generality suppose that $I_{n+1}=0$. Let $I=I_{n}$. Choose $P \varsubsetneqq Q$ with $R / Q$ a $\beta$-G-critical module. Obviously $Q$ is $G$-irreducible and hence $G$-primary. If $Q^{\prime}=\sqrt{Q} \neq Q, \kappa_{G}(R / Q)<\beta$, a contradiction. Hence $Q$ is $G$-prime. Now, $\cap\left(I \cap Q^{(t)}\right) \subseteq \bigcap Q^{(t)}=0$, since $R$ is $G$-primary, and so for infinitely many $t \geq 0$, $\operatorname{I\cap } Q^{(t)} / I_{n} \cap Q^{(t+1)} \neq 0$, otherwise some power of $Q$ is zero. Therefore each nonzero factor $I_{n} \cap Q^{(t)} / I_{n} \cap Q^{(t+1)}$ has Krull dimension equal to $\beta$ by induction. Thus $\kappa_{G}\left(I_{n}\right)>\beta$ by induction.

Corollary 3. Let I be a $G$-ideal of $R$. Then $I$ is $G$-prime if and only if $R / I$ is $G$-critical.

Proof. If $I$ is $G$-prime then the lemma shows that $R / I$ is $G$-critical. Conversely, suppose that $R / I$ is $G$-critical. Certainly $I$ is $G$-irreducible and hence $G$-primary. If $P=\sqrt{I} \neq I$ then by the lemma $\kappa_{G}(R / P)=\kappa_{G}(P / I)$, and this contradicts the fact that $R / I$ is $G$-critical. Thus $I=P$ whence $I$ is $G$-prime.

Proof of Theorem 2. First we prove that $d_{G}(R) \leq \kappa_{G}(R)$. Assume the result for proper $G$-factors of $R$. Then $R$ is $G$-prime, otherwise a contradiction is easily obtained. Let $\kappa_{G}(R)=\alpha$ and let $P$ be any nonzero $G$-prime of $R$. Then by induction, and part (i) of the lemma, $d_{G}(R / P) \leq \kappa_{G}(R / P)<\kappa_{G}(R)$. Hence $d_{G}(R) \leq \kappa_{G}(R)$. Conversely, we prove that $\kappa_{G}(R) \leq d_{G}(R)$. If $\kappa_{G}(R)=\alpha$ then there is a $G$-prime ideal $I$ such that $R / I$ is $\alpha$ - $G$-critical and so $I$ is $G$-prime. Thus, assume $R$ is $G$-prime, and that the result holds for proper $G$-factors of $R$. Let $\beta<\alpha$ and choose a $G$-prime $P$ with $\kappa_{G}(R / P)=\beta$. By induction, $d_{G}(R / P) \geq \beta$ and hence $d_{G}(R)>d_{G}(R / P) \geq \beta$ for any $\beta<\alpha$. Thus $d_{G}(R) \geq \alpha$.

\section{REFERENCES}

1. J. C. McConnell and J. C. Robson, Noncommutative Noetherian rings (John Wiley, 1987).

2. J. E. Roseblade, Prime ideals in group rings of polycyclic groups, Proc. London Math. Soc. (3) 36 (1978), 385-477.

Department of Mathematics

Virginia Polytechnic Institute and State University

BlacksBurg, Virginia 24061-0123 